\title{
Residual kidney function in twice-weekly hemodialysis: irreplaceable contribution to dialysis adequacy
}

\author{
Yu-Ji Lee ${ }^{1,2}$, Connie M. Rhee ${ }^{1}$, Kamyar Kalantar-Zadeh ${ }^{1,3,4}$ \\ ${ }^{1}$ Harold Simmons Center for Kidney Disease Research and Epidemiology, Division of Nephrology \& Hypertension, University of California Irvine \\ Medical Center, Orange, CA, USA; ${ }^{2}$ Division of Nephrology, Department of Medicine, Samsung Changwon Hospital, Sungkyunkwan University \\ School of Medicine, Changwon, Korea; ${ }^{3}$ Nephrology Section, Tibor Rubin Veterans Affairs Medical Center, Long Beach, CA, USA; ${ }^{4}$ Department of \\ Epidemiology, UCLA Fielding School of Public Health, Los Angeles, CA, USA \\ Correspondence to: Kamyar Kalantar-Zadeh, MD, MPH, PhD. Harold Simmons Center for Kidney Disease Research, Division of Nephrology and \\ Hypertension, University of California Irvine Medical Center, Orange, CA, USA. Email: kkz@uci.edu. \\ Comment on: Leong SC, Sao JN, Taussig A, et al. Residual Function Effectively Controls Plasma Concentrations of Secreted Solutes in Patients on \\ Twice Weekly Hemodialysis. J Am Soc Nephrol 2018;29:1992-9.
}

Submitted Jun 28, 2018. Accepted for publication Jul 16, 2018.

doi: 10.21037/atm.2018.07.17

View this article at: http://dx.doi.org/10.21037/atm.2018.07.17

Over time, there has been increasing recognition of the importance of preservation of residual kidney function (RKF) in dialysis patients (1). The presence of RKF has been shown to contribute to the overall health and wellbeing of dialysis patients including improved survival, better nutrition, and reduced inflammation (2-4). Compared to patients receiving peritoneal dialysis, the importance of RKF among patients receiving hemodialysis has been relatively less appreciated and mostly disregarded at the commencement of hemodialysis, given prior reports that RKF declines more rapidly following hemodialysis initiation $(5,6)$. However, contrary to popular belief, rapid decline of RKF does not necessarily occur after transitioning to hemodialysis, although those who are receiving this modality may be at risk of RKF loss owing to potential intradialytic hypotension, nephrotoxic drugs, and coexisting comorbidities (e.g., congestive heart failure) (7). Moreover, given growing evidence demonstrating robust associations between RKF and survival in hemodialysis patients, preservation of RKF has become a valid therapeutic goal in this population $(2,8)$.

Traditionally, the thrice-weekly hemodialysis start has been considered as the standard treatment regimen for initiation of therapy among incident hemodialysis patients regardless of their RKF. It is not clear, however, why dialysis should be "started" abruptly, as opposed to a gradual "transition" to dialysis, using approaches such as less frequent dialysis treatment including twice-weekly hemodialysis (9). In the English language, there are indeed fundamental differences in meaning between the terminology "start" vs. "transition" (see Figure 1). Based on the 2006 Kidney Disease Outcome Quality Initiative (KDOQI) Clinical Practice Guidelines, Guideline 4.3.2., twice-weekly hemodialysis is not appropriate for patients with renal urea clearance $(\mathrm{Kr})<2 \mathrm{~mL} / \mathrm{min} / 1.73 \mathrm{~m}^{2}$, while the Work Group advised that thrice-weekly hemodialysis as a minimum frequency level was no longer appropriate (10). Based on solute kinetics, the KDOQI Work Group was comfortable recommending a twice-weekly dialysis schedule among patients with substantial RKF, defined as those with a $\mathrm{Kr}>3 \mathrm{~mL} / \mathrm{min}$ to be initiated on twice-weekly hemodialysis, and those with a $\mathrm{Kr}>2 \mathrm{~mL} / \mathrm{min}$ who will maintain this regimen (see Figure 2).

Recently, several observational studies have shown that twice-weekly hemodialysis is associated with better preservation of RKF and higher health-related quality of life $(11,12)$. These findings have sparked greater interest in considering twice-weekly hemodialysis as an initial dialysis prescription. In addition, twice-weekly hemodialysis has demonstrated similar survival rates compared to that of 


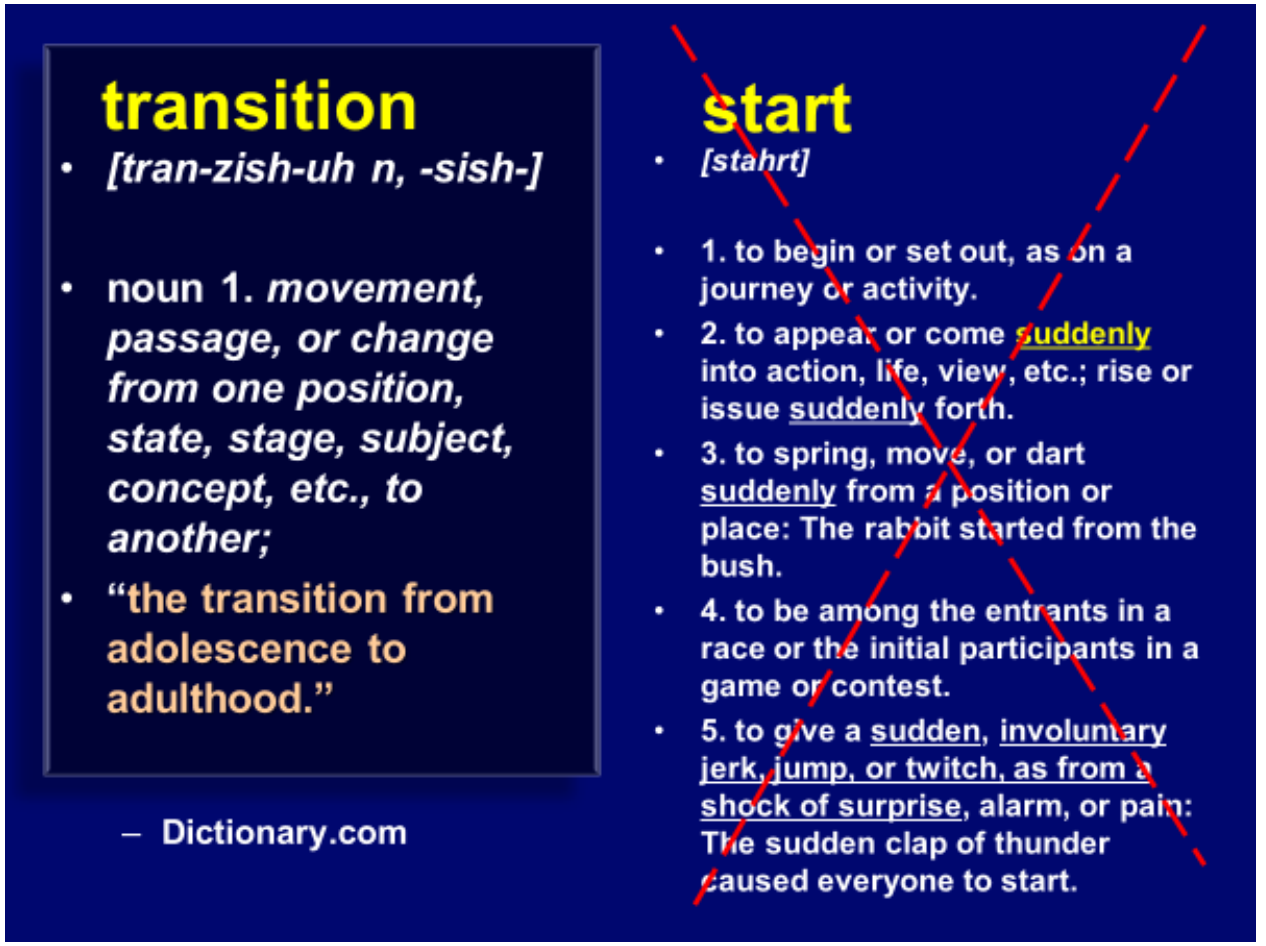

Figure 1 Comparing the definitions of "start" versus "transition" in order to better understand the concepts of "starting" dialysis abruptly as thrice-weekly hemodialysis versus incrementally "transitioning" to dialysis as twice-weekly hemodialysis (9).

\section{KDOQI Guidelines recommends twice-weekly (2x/wk) hemodialysis if $\mathrm{Kr}$ (residual kidney function) is $>3 \mathrm{ml} / \mathrm{min}$ and maintaining $2 x / w k H D$ as long as $\mathrm{Kr}>2 \mathrm{ml} / \mathrm{min}$}

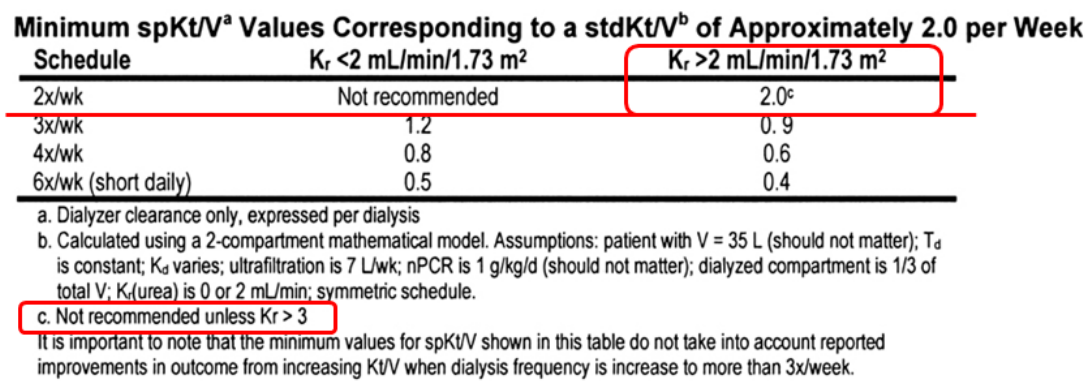

With a regular dialysis dose of $\mathrm{Kt} / \mathrm{N}_{\mathrm{sp}}$ of $1.2, \mathrm{Kr}>3$ $\mathrm{mL} / \mathrm{min} / 1.73 \mathrm{~m}^{2}$ would be required to meet the minimum $\mathrm{Kt} / \mathrm{V}$ with $2 \mathrm{x} / \mathrm{wk} \mathrm{HD}$.

Figure 2 The KDOQI guidelines pertaining to the recommended frequency of hemodialysis treatment per week based on residual kidney function defined by renal urea clearance $(\mathrm{Kr})(10)$. 
thrice-weekly hemodialysis among patients with substantial RKF (11). Given these findings, clinical practice guidelines allowed for the use of twice-weekly hemodialysis among patients with substantial RKF and have recently increased the weight of $\mathrm{RKF}$ incorporated into dialyzer $\mathrm{Kt} / \mathrm{V}$ to calculate standard $\mathrm{Kt} / \mathrm{V}_{\text {urea }}(13)$.

The beneficial impact of RKF on patient outcomes may be related to improved volume control and greater solute clearance, particularly that of middle molecules and protein-bound solutes $(14,15)$. Traditionally, $\mathrm{Kt} / \mathrm{V}_{\text {urea, }}$ small solute clearance has been the standard metric for measuring dialysis adequacy. However, higher dialyzer urea clearance (high-dose $\mathrm{Kt} / \mathrm{V}_{\text {urea }}$ ) failed to improve patient outcomes in the Hemodialysis (HEMO) study (16). This may be in part due to inadequate removal of uremic solutes other than urea by hemodialysis alone. Furthermore, the characteristics of solute clearance offered by dialysis $v s$. RKF are different. For example, protein-bound solutes are largely secreted by organic acid transporters in the proximal renal tubule, and so removed by the kidney (17). One concern regarding the prescription of twice-weekly hemodialysis has been the possibility of providing less solute clearance due to the longer interdialytic interval and reduced treatment frequency. Yet when considering the beneficial effects of RKF, twice-weekly hemodialysis may not be inferior in patients with substantial RKF compared to thrice-weekly hemodialysis in patients without RKF. However, from the viewpoint of dialysis adequacy, there are ongoing knowledge gaps with respect to how RKF contributes to solute clearance as compared with hemodialysis among patients receiving twice-weekly treatment.

In a recent issue of the Fournal of the American Society of Nephrology, Leong et al. has addressed the contribution of RKF to the removal of secreted solutes in twice-weekly hemodialysis patients (18). In this study, the investigators measured plasma concentrations, residual renal clearance, and dialytic clearance for urea and secreted solutes including hippurate, phenylacetylglutamine, indoxyl sulfate, and p-cresol sulfate in nine patients with RKF on twice-weekly hemodialysis and nine anuric patients on thrice-weekly hemodialysis, respectively. Averaged generation rates and dialytic clearance of secreted solutes and urea clearance (standard $\mathrm{Kt} / \mathrm{V}_{\text {urea }}$ ) were similar in both groups, but the plasma concentrations of hippurate and phenylacetylglutamine were significantly lower in patients with RKF on twice-weekly hemodialysis in comparison to anuric patients on thrice-weekly hemodialysis. Although the dialytic clearances of these solutes were lower compared to the dialytic clearance of urea, the renal clearances of these solutes were significantly higher compared to the renal clearance of urea. On the other hand, both dialytic and renal clearances of indoxyl sulfate and p-cresol sulfate were lower compared to those of urea, probably because of the stronger protein-binding capacity of these solutes. Nonetheless, fractions of these solutes removed by renal clearance were still higher than that of urea removed by renal clearance. Interestingly, even though patients on twice-weekly hemodialysis had lower ultrafiltration volumes, slower dialysate flow rates, and shorter dialysis times per session, they had similar plasma levels of urea, indoxyl sulfate, and p-cresol sulfate, and even lower plasma levels of hippurate and phenylacetylglutamine compared to those on thriceweekly hemodialysis.

Despite the small sample size of the study, these important findings reported by Leong et al. are generally consistent with those of previous studies and support the view that RKF contributes more importantly to the removal of secreted solutes as compared with dialysis $(15,19)$. Furthermore, the authors concluded that plasma levels of these solutes can be well controlled by twice-weekly hemodialysis in patients with RKF to the same degree as thrice-weekly hemodialysis in anuric patients. However, the relative importance of RKF on clearance rates can vary among secreted solutes; it was greater for hippurate and phenylacetylglutamine than for indoxyl sulfate and p-cresol sulfate in this study. The variability of residual clearance for different secreted solutes has been observed in previous studies (20). This may be explained by the differential degree of protein binding, reduced availability for transporters, and biologic variability in protein expression of transporters among different solutes.

Although the clearance of middle molecules such as $\beta 2$ microglobulin may be enhanced by some hemodialysis techniques, increasing treatment adequacy or frequency of hemodialysis is largely limited or ineffective in improving the clearance of secreted solutes $(16,21,22)$. Moreover, these approaches may potentially lead to more accelerated decline in RKF (23). Hence, preserving RKF in hemodialysis 
patients may be more effective in removing secreted solutes as opposed to increasing the frequency of hemodialysis. Furthermore, the continuous nature of RKF, even in small amounts, may have a greater impact on total solute clearance in comparison to the intermittent nature of hemodialysis.

Following the study by Leong et al., it still remains unknown as to whether greater removal of certain secreted solutes by RKF among twice-weekly hemodialysis patients has clinical relevance. While indoxyl sulfate and p-cresol sulfate are among the most commonly studied secreted solutes in dialysis patients, in this study plasma concentrations of these solutes did not differ between patients with RKF on twice-weekly hemodialysis $v s$. those without RKF on thrice-weekly hemodialysis. Indeed, plasma concentrations of protein-bound solutes such as indoxyl sulfate and $\mathrm{p}$-cresol sulfate have showed wide variation among patients in previous studies $(15,21)$. Differences in $\mathrm{Kt} / \mathrm{V}_{\text {urea }}$ and even in RKF accounted for little of this variation in plasma levels of protein-bound solutes. As protein-bound solutes are synthesized by the gut flora, the generation rates of these solutes varied considerably among hemodialysis patients and were not closely associated with $\operatorname{RKF}(15,17)$. In addition to the variation in solute generation rates, distribution volumes and the non-renal clearances of different solutes may also influence plasma concentration of secreted solutes. Subsequently, these factors may obscure the ability to discern the influence of RKF on plasma concentrations of protein-bound solutes. Moreover, the study by Leong et al. assessed only four protein-bound solutes, and thus could not assess the impact of RKF on other uremic solutes among patients on twiceweekly hemodialysis. Finally, the aforementioned study did not assess clinical outcomes among patients with RKF prescribed twice-weekly hemodialysis $v s$. those who were anuric on thrice-weekly hemodialysis. In an analysis of the HEMO study cohort who largely had little to no RKF, hippurate, p-cresol sulfate, indoxyl sulfate, and phenylacetylglutamine concentrations were not associated with cardiovascular outcomes (24). However, some studies have suggested that uremic toxicity and adverse clinical outcomes may ensue from these solutes $(17,25)$. Therefore, an adequately powered clinical trial is needed to determine whether twice-weekly hemodialysis among patients with higher RKF will result in greater preservation of RKF and improved patient outcomes beyond that of reduced concentrations of circulating secreted solutes. In addition, further studies are needed to identify adjuvant strategies that will best preserve residual function among patients with substantial RKF who initiate twice-weekly hemodialysis.

In summary, the study by Leong et al. provides reassuring evidence that twice-weekly hemodialysis in patients with RKF is associated with similar plasma concentrations of secreted solutes and near-equivalent dialysis adequacy as thrice-weekly hemodialysis in anuric patients. As discussed by the investigators, these results may affirm recently updated guidelines that have given greater weight to RKF in calculating total clearance and adequacy, and may also inspire further studies that incorporate assessment of RKF in the prescription of dialysis.

\section{Acknowledgements}

None.

\section{Footnote}

Conflicts of Interest: The authors have no conflicts of interest to declare.

\section{References}

1. Wang AY, Lai KN. The importance of residual renal function in dialysis patients. Kidney Int 2006;69:1726-32.

2. Termorshuizen F, Dekker FW, van Manen JG, et al. Relative contribution of residual renal function and different measures of adequacy to survival in hemodialysis patients: an analysis of the Netherlands Cooperative Study on the Adequacy of Dialysis (NECOSAD)-2.J Am Soc Nephrol 2004;15:1061-70.

3. Pecoits-Filho R, Heimburger O, Barany P, et al. Associations between circulating inflammatory markers and residual renal function in CRF patients. Am J Kidney Dis 2003;41:1212-8.

4. Suda T, Hiroshige K, Ohta T, et al. The contribution of residual renal function to overall nutritional status in chronic haemodialysis patients. Nephrol Dial Transplant 2000;15:396-401.

5. Jansen MA, Hart AA, Korevaar JC, et al. Predictors of the rate of decline of residual renal function in incident dialysis 
patients. Kidney Int 2002;62:1046-53.

6. Moist LM, Port FK, Orzol SM, et al. Predictors of loss of residual renal function among new dialysis patients. J Am Soc Nephrol 2000;11:556-64.

7. McKane W, Chandna SM, Tattersall JE, et al. Identical decline of residual renal function in high-flux biocompatible hemodialysis and CAPD. Kidney Int 2002;61:256-65.

8. Obi Y, Rhee CM, Mathew AT, et al. Residual Kidney Function Decline and Mortality in Incident Hemodialysis Patients. J Am Soc Nephrol 2016;27:3758-68.

9. Kalantar-Zadeh K, Kovesdy CP, Streja E, et al. Transition of care from pre-dialysis prelude to renal replacement therapy: the blueprints of emerging research in advanced chronic kidney disease. Nephrol Dial Transplant 2017;32:ii91-8.

10. Hemodialysis Adequacy 2006 Work Group. Clinical practice guidelines for hemodialysis adequacy, update 2006. Am J Kidney Dis 2006;48 Suppl 1:S2-90.

11. Obi Y, Streja E, Rhee CM, et al. Incremental Hemodialysis, Residual Kidney Function, and Mortality Risk in Incident Dialysis Patients: A Cohort Study. Am J Kidney Dis 2016;68:256-65.

12. Bieber B, Qian J, Anand S, et al. Two-times weekly hemodialysis in China: frequency, associated patient and treatment characteristics and Quality of Life in the China Dialysis Outcomes and Practice Patterns study. Nephrol Dial Transplant 2014;29:1770-7.

13. National Kidney F. KDOQI Clinical Practice Guideline for Hemodialysis Adequacy: 2015 update. Am J Kidney Dis 2015;66:884-930.

14. Konings CJ, Kooman JP, Schonck M, et al. Fluid status in CAPD patients is related to peritoneal transport and residual renal function: evidence from a longitudinal study. Nephrol Dial Transplant 2003;18:797-803.

Cite this article as: Lee YJ, Rhee CM, Kalantar-Zadeh $\mathrm{K}$. Residual kidney function in twice-weekly hemodialysis: irreplaceable contribution to dialysis adequacy. Ann Transl Med 2018;6(16):317. doi: 10.21037/atm.2018.07.17
15. Marquez IO, Tambra S, Luo FY, et al. Contribution of residual function to removal of protein-bound solutes in hemodialysis. Clin J Am Soc Nephrol 2011;6:290-6.

16. Eknoyan G, Beck GJ, Cheung AK, et al. Effect of dialysis dose and membrane flux in maintenance hemodialysis. $\mathrm{N}$ Engl J Med 2002;347:2010-9.

17. Neirynck N, Vanholder R, Schepers E, et al. An update on uremic toxins. Int Urol Nephrol 2013;45:139-50.

18. Leong SC, Sao JN, Taussig A, et al. Residual Function Effectively Controls Plasma Concentrations of Secreted Solutes in Patients on Twice Weekly Hemodialysis. J Am Soc Nephrol 2018;29:1992-9.

19. Bammens B, Evenepoel P, Verbeke K, et al. Time profiles of peritoneal and renal clearances of different uremic solutes in incident peritoneal dialysis patients. Am J Kidney Dis 2005;46:512-9.

20. Suchy-Dicey AM, Laha T, Hoofnagle A, et al. Tubular Secretion in CKD. J Am Soc Nephrol 2016;27:2148-55.

21. Meyer TW, Sirich TL, Fong KD, et al. Kt/Vurea and Nonurea Small Solute Levels in the Hemodialysis Study. J Am Soc Nephrol 2016;27:3469-78.

22. Sirich TL, Fong K, Larive B, et al. Limited reduction in uremic solute concentrations with increased dialysis frequency and time in the Frequent Hemodialysis Network Daily Trial. Kidney Int 2017;91:1186-92.

23. Daugirdas JT, Greene T, Rocco MV, et al. Effect of frequent hemodialysis on residual kidney function. Kidney Int 2013;83:949-58.

24. Shafi T, Sirich TL, Meyer TW, et al. Results of the HEMO Study suggest that p-cresol sulfate and indoxyl sulfate are not associated with cardiovascular outcomes. Kidney Int 2017;92:1484-92.

25. Cao XS, Chen J, Zou JZ, et al. Association of indoxyl sulfate with heart failure among patients on hemodialysis. Clin J Am Soc Nephrol 2015;10:111-9. 